\title{
Simulations of resonant Alfvén waves generated by artificial HF heating of the auroral ionosphere
}

\author{
D. Pokhotelov, W. Lotko, and A. V. Streltsov \\ Thayer School of Engineering, Dartmouth College, Hanover, NH 03755, USA \\ Received: 18 August 2003 - Revised: 29 February 2004 - Accepted: 28 May 2004 - Published: 7 September 2004
}

\begin{abstract}
Numerical two-dimensional two-fluid MHD simulations of dynamic magnetosphere-ionosphere (MI) coupling have been performed to model the effects imposed on the auroral ionosphere by a powerful HF radio wave transmitter. The simulations demonstrate that modifications of the ionospheric plasma temperature and recombination due to artificial heating may trigger the ionospheric feedback instability when the coupled MI system is close to the state of marginal stability. The linear dispersion analysis of MI coupling has been performed to find the favorable conditions for marginal stability of the system. The development of the ionospheric feedback instability leads to the generation of shear Alfvén waves which resonate in the magnetosphere between the heated ionospheric E-region and the strong gradient in the Alfvén speed at altitudes of $1-2 R_{E}$. The application of the numerical results for the explanation of observations performed by low-orbiting satellites above the highlatitude ionosphere heated with a high power ground-based HF transmitter is discussed.
\end{abstract}

Key words. Magnetospheric physics (auroral phenomena; magnetosphere-ionosphere interactions; MHD waves and instabilities)

\section{Introduction}

A number of experiments with high frequency (HF) radio wave transmitters have demonstrated that the artificial heating of the auroral ionosphere with radio waves can lead to dramatic changes in the ionospheric parameters. Under favorable conditions the dynamic variations of the ionospheric parameters induced by the HF heater may initiate the ionospheric feedback instability, the process in which the ionosphere plays the role of a driver for magnetospheric ULF pulsations.

As proposed by Atkinson (1970) and Sato (1978), the feedback instability arises when a localized perturbation in ionospheric conductivity become polarized in the presence

Correspondence to: A. Streltsov

(streltsov@dartmouth.edu) of the convection electric field, thus locally reducing the ionospheric Joule dissipation. Energy released by this process radiates into the magnetosphere in the form of shear Alfvén waves. After reflection from the conjugate ionosphere or magnetospheric plasma inhomogeneities, the stimulated Alfvén wave returns to the ionosphere, further modifying the initial conductivity perturbation. The feedback instability can simultaneously generate field-line eigenmodes standing along the entire magnetic field line between two conjugate ionospheres and shorter-period resonance Alfvén waves trapped between the ionosphere and the Alfvén speed peak at the altitudes of $1-2 R_{E}$ (e.g. Pokhotelov et al., 2002a). The initial ionospheric perturbation can arise naturally due to the auroral electron precipitation or, alternatively, it can be induced artificially by means of ionospheric heating (Trakhtengertz et al., 2000).

Effects imposed on the ionosphere by a powerful HF heater can be summarized as follows. The oscillating electric field of radio waves increases the electron temperature in the affected region of the ionosphere. Within the ionospheric E-layer, between about $80 \mathrm{~km}$ and $120 \mathrm{~km}$ altitude, enhancement of the electron temperature results in the reduction of the plasma recombination coefficient that ultimately leads to the enhancement of plasma density. However, above the altitude of $200 \mathrm{~km}$, the plasma transport processes start to be more significant when comparing the changes in plasma recombination. As a consequence, in the ionospheric F-layer, the enhancement of electron temperature due to HF radio waves causes a depletion in plasma density (Gurevich, 1978; Stocker et al., 1997).

Experiments at the EISCAT heating facility in Northern Scandinavia have shown that the plasma temperature and density within the heated region of the ionosphere may change by $15-25 \%$ of its ambient value (Stocker et al., 1992; 1997). Even much larger variation in temperatures of the ionospheric electrons $(200 \%-300 \%)$ and ions $\left(100^{\circ}\right.$ above background) were reported by Rietveld et al. (2003). These changes lead to the modification of ionospheric conductivity that in turn affects the ionospheric current closure. In a series of EISCAT experiments (Stubbe et al., 1982; 1985) the HF heating signal has been modulated in the ULF frequency 
range leading to the generation of ULF magnetic pulsations. However, the amplitude of the stimulated ULF pulsations, registered by ground-based magnetometers, turned out to be almost two orders of magnitude higher than predicted by the theory. To explain this discrepancy between theory and observations, Stubbe et al. (1982) suggest that the ionospheric feedback instability may be responsible for the observed wave amplification.

In a recent experimental study of artificial heating effects on the auroral ionosphere, Robinson et al. (2000) used the EISCAT HF transmitter to heat an ionospheric spot $25 \mathrm{~km}$ in diameter. During the time of this experiment the FAST satellite flew over the heated ionospheric region in a nearly meridional traversal at the altitude of $2550 \mathrm{~km}$. The satellite registered disturbances in the perpendicular electric field with an amplitude of about $5-10 \mathrm{mV} / \mathrm{m}$ and with a frequency of the heater modulation $(3 \mathrm{~Hz})$ above the heated spot. These measurements were evidently accompanied by downward fluxes of electrons with energies of $50-150 \mathrm{eV}$. The intensification of discrete aurora stimulated by the artificial heating of the ionospheric E-layer in the pre-midnight sector has been observed in a separate EISCAT heating experiment by Blagoveshchenskaya et al. (2001). Authors of both experiments suggested that the observed electromagnetic disturbances and the auroral precipitation associated with them were produced by the feedback instability inside the resonance cavity, formed by the ionospheric E-layer and peak in the Alfvén velocity. This is the so-called ionospheric Alfvén resonator (IAR) first introduced by Polyakov and Rapoport (1981) and extensively studied after that (e.g. Trahtengertz and Feldstein, 1991; Lysak, 1991; Pokhotelov, 2000; Lysak and Song, 2002).

This paper presents results from a numerical study of how the feedback instability can be initiated inside the IAR by an artificial heating of the auroral ionosphere when the magnetosphere-ionosphere (MI) system is close to a state of marginal stability. The dispersion analysis of the ionospheric feedback instability has been performed in order to find the conditions for marginal stability. The study is based on a two-dimensional numerical model describing dispersive shear Alfvén waves in a strongly inhomogeneous magnetospheric plasma. The ionospheric feedback mechanism is included in the model via active ionospheric boundary conditions.

\section{Model of the active auroral ionosphere}

The behavior of the active auroral ionosphere has been modelled using the current and density continuity equations. The ionospheric current continuity equation can be expressed in the height-integrated form as

$\nabla_{\perp} \cdot\left(\Sigma_{P} \boldsymbol{E}_{\perp}\right)=-j_{\|} \cdot$

Here the subscripts $\|$ and $\perp$ denote vector components in the directions parallel and perpendicular to the background magnetic field, respectively; the subscripts " 0 " and " 1 " denote background (time independent) and perturbed values of the physical parameters, respectively; $\boldsymbol{E}_{\perp}=\boldsymbol{E}_{\perp 0}+\boldsymbol{E}_{\perp 1}$ is the convection electric field at the ionosphere; $\Sigma_{P}$ is the heightintegrated ionospheric Pedersen conductivity; and $j_{\|}$is the magnitude of field-aligned current density. The ionospheric Pedersen conductivity is given by $\Sigma_{P}=e h M_{p}\left(n_{0}+n_{1}\right)$, where $e$ is the electron charge; $h=20 \mathrm{~km}$ is the effective thickness of the ionospheric conducting layer; $n_{0}$ and $n_{1}$ are background and perturbed parts of the plasma density, respectively; and $M_{P}$ denotes the ion Pedersen mobility.

The plasma density continuity equation for the auroral ionosphere is given by

$\frac{\partial n_{1}}{\partial t}=\frac{j_{\|}}{e h}+\mathcal{S}-\alpha n^{2}$,

where $n=n_{0}+n_{1}$ is the plasma number density; $\mathcal{S}=\alpha n_{0}^{2}$ is an ionization source due to the solar radiation and a steady precipitation of hot plasma particles from the magnetosphere that maintains an equilibrium density $n_{0}$; and $\alpha$ is the plasma recombination coefficient.

\section{MHD model of the magnetosphere}

MHD equations describing the propagation of shear Alfvén waves in the warm inhomogeneous magnetospheric plasma includes (e.g. Streltsov and Lotko, 1998) the electron parallel momentum equation

$m_{e} n_{0}\left(\frac{\partial v_{\| e}}{\partial t}+v_{e} v_{\| e}\right)+e n_{0} E_{\|}+\hat{\boldsymbol{b}} \cdot \nabla p_{e}=0$

the plasma density continuity equation

$\frac{\partial n_{1}}{\partial t}+\nabla \cdot n_{0} v_{\| e} \hat{\boldsymbol{b}}=0$

and the current continuity equation

$$
\begin{aligned}
&\left(1-\rho_{i}^{2} \nabla_{\perp}^{2}\right) \nabla \cdot j_{\|} \hat{\boldsymbol{b}}+ \\
& \frac{1}{\mu_{o}} \nabla \cdot\left(\frac{1}{v_{A}^{2}}+\frac{1-\rho_{i}^{2} \nabla_{\perp}^{2}}{c^{2}}\right) \frac{\partial \boldsymbol{E}_{\perp}}{\partial t}=0 .
\end{aligned}
$$

Here $v_{\| e}$ and $p_{e}$ are the electron parallel speed and pressure; $m_{e}$ is the electron mass; $\hat{\boldsymbol{b}}$ is a unit vector in the direction of the ambient magnetic field; $v_{A}$ is the Alfvén speed; $\rho_{i}$ is the ion Larmour radius; $c$ is the speed of light and $\mu_{0}$ is the permeability of free space.

Enhanced collisions of electrons with ions and neutrals inside the ionosphere lead to the appearance of a parallel collisional resistivity, described by the collision frequency $v_{e}$ in the electron parallel momentum equation. The parallel resistivity of the upper ionosphere slows the field-aligned electron motion which leads to the suppression of small-scale fieldaligned currents and the generation of parallel electric fields at low altitudes (Forget et al., 1991; Borovsky, 1993). In simulations collisional resistivity imposes a physical limit on the smallest transverse wavelength of the numerical solutions. 


\section{Dispersion analysis of the feedback instability}

In order to determine the conditions for marginal stability of the coupled magnetosphere-ionosphere system, a dispersion analysis of the ionospheric feedback instability has been performed considering the magnetosphere as a simplified, lumped transmission line. Neglecting the curvature of magnetic field lines and assuming the Alfvén speed to be constant along the field lines, the equation of magnetospheric response to ionospheric electric field perturbation $\boldsymbol{E}_{\perp 1}$ can be expressed in a form given by Miura and Sato (1980):

$\frac{2 \mu_{0} l}{\pi^{2}} \frac{\partial j_{\|}}{\partial t}+\left(\left(\frac{l}{\pi v_{A l}}\right)^{2} \frac{\partial^{2}}{\partial t^{2}}+1\right) \nabla_{\perp} \cdot \boldsymbol{E}_{\perp 1}=0$,

where $l$ is the length of magnetic field line and $v_{A l}$ is the mean value of Alfvén speed.

Equation (6), combined with linearized equations for the ionospheric current continuity Eq. (1) and density continuity Eq. (2) leads to the dispersion relation expressed by the third-order complex polynomial given in the Appendix. The numerical solution of this dispersion equation (Eq. (A1)) is presented in Fig. 1, where the color intensity shows the amplitude of the feedback instability growth rate for different values of ionospheric parameters. Parameters describing the magnetosphere in Eq. (6) have been chosen as $l=2 R_{E}$ and $v_{A l}=5000 \mathrm{~km} / \mathrm{s}$ which sustain a typical eigenperiods of IAR defined by Polyakov and Rapoport (1981) and Lysak (1991).

The top panel in Fig. 1 corresponds to the case when $E_{\perp 0}$ and $\alpha$ have been taken to be constant while $k_{\perp}$ and $\Sigma_{P 0}$ vary. Values of the recombination coefficient and background electric field have been taken as $\alpha=3 \cdot 10^{-7} \mathrm{~cm}^{3} / \mathrm{s}$ (Nygrén et al., 1992) and $E_{\perp 0}=50 \mathrm{mV} / \mathrm{m}$ (Kelley, 1989), and the range of background Pedersen conductivity variations has been chosen according to Hardy et al. (1987). The central panel in Fig. 1 corresponds to the case when the wave number and recombination coefficient have been fixed as $k_{\perp}=2.5 \mathrm{~km}^{-1}$ and $\alpha=3 \cdot 10^{-7} \mathrm{~cm}^{3} / \mathrm{s}$, respectively, while the transverse wave number and Pedersen conductance vary. The bottom panel in Fig. 1 represents the case when $k_{\perp}$ and $E_{\perp 0}$ are fixed. A black line shows where the instability growth rate, $\Gamma$, is equal to zero.

The dispersion analysis demonstrates that the growth rate of the feedback instability accelerates under conditions of low ionospheric conductance and strong electric convection. Increasing the plasma recombination coefficient $\alpha$ reduces the growth rate of the instability. Results of the linear dispersion analysis have been used to determine the ionospheric parameters corresponding to the state of marginal stability of the MI system. These parameters have been used for a numerical modeling of artificial heating of the auroral ionosphere described in the next section. It is noted that the dispersion analysis can only provide approximate criteria for marginal stability, since the dispersion relation Eq. (A1) is derived using the oversimplified lumped transmission line model of the magnetosphere Eq. (6), which does not take into account the effects of parallel inhomogeneities of the magnetosphere and ionospheric collisional resistivity. A more
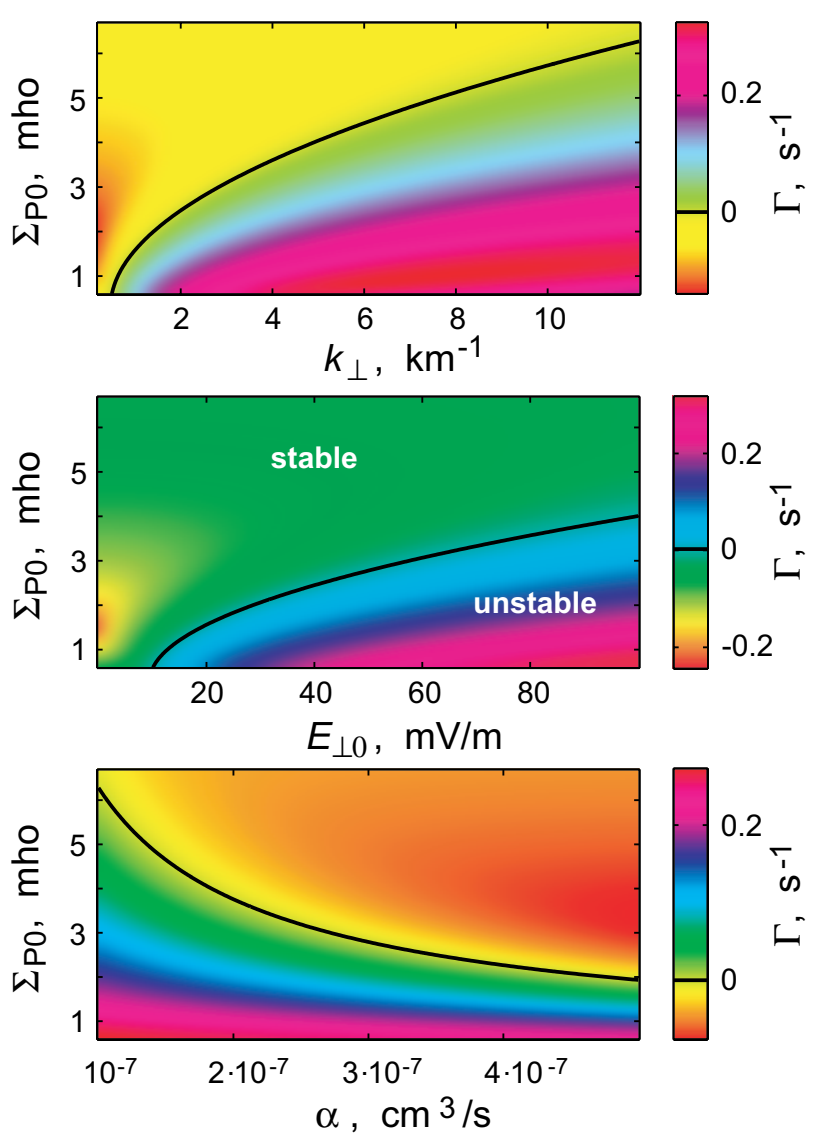

Fig. 1. (Top) Time variations of the ionospheric plasma recombination coefficient, $\alpha$, (solid line) and the electron collision frequency, $v_{e}$, (dashed line). (Bottom) Time variations of the field-aligned current density, $j_{\|}$measured at the ionosphere at $L=6.2$ when the heater is turned off all the time (thick line) and when the heater is turned on after $t=40 \mathrm{~s}$ (thin line).

accurate stability analysis requires numerical simulations as described below.

\section{Numerical heating experiment}

A numerical model of the active auroral ionosphere, combined with a two-fluid MHD model of the magnetospheric dispersive Alfvén dynamics has been used to simulate the effects imposed on the ionosphere by a HF heater. The coupled system of ionospheric Eqs. (1)-(2) and two-fluid magnetospheric MHD Eqs. (3)-(5) has been solved numerically in two-dimensional dipolar coordinates $(L, \mu)$ (e.g. Streltsov and Lotko, 1997), representing the magnetospheric flux tube between magnetic shells $L_{1}=6$ and $L_{2}=6.4$ with the HF heater centered at $L=6.2$. A finite difference method has been applied to solve numerically the system of differential Eqs. (1)-(5). Detailed description of the numerical technics can be found in Streltsov and Lotko (1997) and Pokhotelov et al. (2002a, b). 

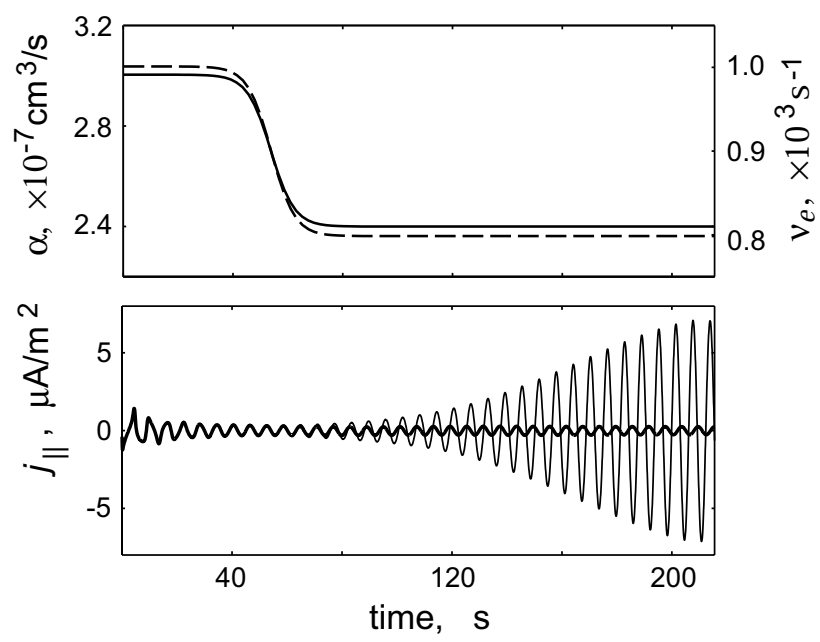

Fig. 2. (Top) Time variations of the ionospheric plasma recombination coefficient, $\alpha$, (solid line) and the electron collision frequency, $v_{e}$, (dashed line). (Bottom) Time variations of the field-aligned current density, $j_{\|}$measured at the ionosphere at $L=6.2$ when the heater is turned off all the time (thick line) and when the heater is turned on after $t=40 \mathrm{~s}$ (thin line).

Utilizing the preceding dispersion analysis, it has been estimated that the magnetosphere-ionosphere system should be close to a state of a marginal stability (slightly below the instability threshold) when the background ionospheric parameters are chosen as follows: $E_{\perp 0}=50 \mathrm{mV} / \mathrm{m}$; $\alpha=3 \cdot 10^{-7} \mathrm{~cm}^{3} / \mathrm{s}$; and $\Sigma_{P 0}=3$ mho. These values have been used as background ionospheric parameters in the numerical simulations. At the poleward and equatorward sides of the domain Dirichlet-type boundary conditions for the background electrostatic potential have been imposed in such a way that they provide a background poleward-directed electric field, homogenous in latitudinal direction, with the magnitude of $E_{\perp 0}=50 \mathrm{mV} / \mathrm{m}$ at the altitudes of ionospheric E-layer. Similar to Pokhotelov et al. (2002a, b), the numerical model includes strong parallel inhomogeneities in the Alfvén speed profile with the values of Alfvén speed reaching $50000 \mathrm{~km} / \mathrm{s}$ at the altitude of $1.5 R_{E}$ comparing to $1200 \mathrm{~km} / \mathrm{s}$ near the ionosphere. The magnitude of the Alfvén speed at low altitudes can be much lower if heavy ions $\left(\mathrm{NO}^{+}\right.$ and $\mathrm{O}_{2}^{+}$) will be taken into the consideration. In the present model the plasma is assumed to be pure hydrogen.

Spatial and temporal variations of the plasma recombination coefficient $\alpha$, due to the electron temperature enhancement in the E-layer, have been modeled as

$$
\begin{gathered}
\alpha=\alpha_{0}\left(1-0.05\left(1+\cos \left(2 \pi \frac{L}{L_{2}-L_{1}}-\pi\right)\right)\right. \\
\left.\cdot\left(1+\tanh \left(8 \pi \frac{t}{t_{0}}-2 \pi\right)\right)\right),
\end{gathered}
$$

where $\alpha_{0}=3 \cdot 10^{-7} \mathrm{~cm}^{3} \mathrm{~s}$ is the background plasma recombination coefficient (Nygrén et al., 1992); and $t_{0}=220 \mathrm{~s}$ is the time length of simulation run. As has been demonstrated by linear dispersion analysis the reduction of plasma recombination coefficient increases the growth rate of feedback in- stability. Time variations of the recombination coefficient at the center of computational domain $L=6.2$ are shown with a solid line in the top panel in Fig. 2.

Since the heating mechanism also affects plasma density in the ionospheric F-layer, the accompanying reduction in the ionospheric electron collision frequency $v_{e}$ has also been included in the model. The reduction in $v_{e}$ reduces the ionospheric collisional resistivity, thus stimulating the growth of the feedback instability. Spatial and temporal changes in the ionospheric collision frequency, due to the HF heating, have been modeled as

$$
\begin{gathered}
\nu_{e}=v_{e 0} e^{-12 r}\left(1-0.05\left(1+\cos \left(2 \pi \frac{L}{L_{2}-L_{1}}-\pi\right)\right)\right. \\
\left.\cdot\left(1+\tanh \left(8 \pi \frac{t}{t_{0}}-2 \pi\right)\right)\right),
\end{gathered}
$$

where $v_{e 0}=10^{3} \mathrm{~s}^{-1}$ is the background value of the ionospheric electron-neutral collision frequency (Kelley, 1989) and $r$ is the distance along the ambient magnetic field from the E-layer measured in the Earth's radii. The dashed line in the top panel in Fig. 2 shows time variations of $v_{e}$ at the center of computational domain due to the heating.

The computations with these background ionospheric parameters have been performed, assuming that the initial ionospheric density perturbation is given by a Gaussian function

$$
\begin{aligned}
n_{1}=0.05 n_{0} & \left(\exp \left(-\frac{\left(L-L_{c}+\delta L / 2\right)^{2}}{(\delta L)^{2}}\right)\right. \\
- & \left.\exp \left(-\frac{\left(L-L_{c}-\delta L / 2\right)^{2}}{(\delta L)^{2}}\right)\right),
\end{aligned}
$$

where $L_{c}=6.2$ and $\delta L=0.2$. Zero Dirichlet-type boundary conditions for perturbed quantities $n_{1}, A_{\|}$and $\phi_{1}$ were imposed along the lateral boundaries of the computational domain.

When the heater is turned off all the time, i.e. $\alpha=\alpha_{0}$ and $v_{e}=v_{e 0} e^{-12 r}$, the system appears to be stable. The thick line in the bottom panel in Fig. 3 shows the amplitude of the fieldaligned current density at the ionosphere at $L=6.2$ as a function of time computed when the heater is off. In another computational run the heater is turned on after the time moment $t=40 \mathrm{~s}$, making the system feedback-unstable. In this case the amplitude of the field-aligned current density starts to grow, as shown with a thin line in the bottom panel in Fig. 2.

Figure 3 shows the amplitude of the transverse electric $E_{\perp 1}$ and magnetic $B_{\perp 1}$ fields within half of the computational domain: between the ionosphere and the magnetic equator. Another half of the computational domain is not shown in the figure since this part of the ionosphere has not been heated and remains stable.

Oscillations of the field-aligned current density with a period of about 6.0-6.5 s, as seen in Fig. 2, are associated with a bouncing of Alfvén waves inside the IAR. Recent simulations by Streltsov and Lotko (2003) show that the frequency of oscillations measured by low-orbiting satellites can be significantly modified by the Doppler shift due to the spacecraft motion and relatively high (of the order of a few $\mathrm{km} / \mathrm{s}$ ) perpendicular phase velocity of the small-scale waves. Figure 4 

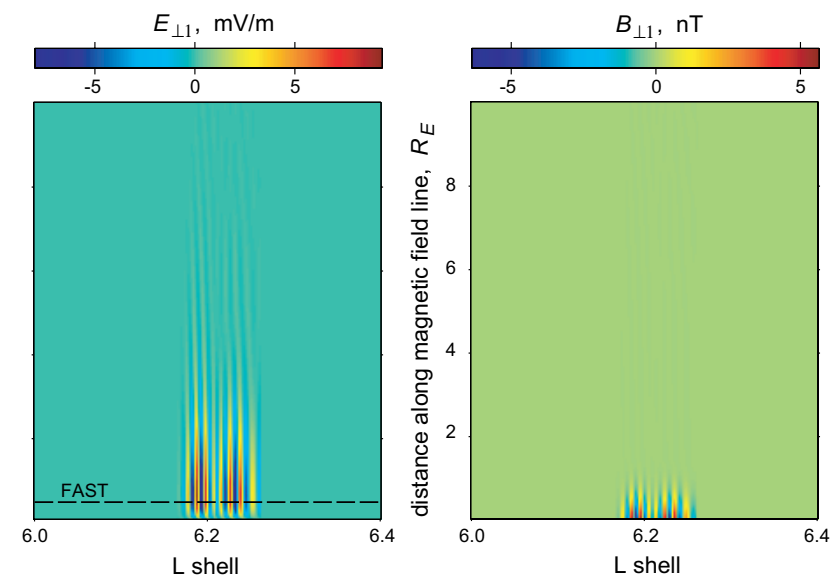

Fig. 3. Snapshot of the transverse electric field, $E_{1 \perp}$, and the transverse magnetic field, $B_{1 \perp}$, inside the half of the computational domain at the time $t=200 \mathrm{~s}$.

shows the transverse electric and magnetic field, and fieldaligned current density observed by a virtual satellite orbiting with a speed of $5 \mathrm{~km} / \mathrm{s}$ above the heated region of the ionosphere. The virtual satellite was flying across the computational domain at $2500 \mathrm{~km}$ orbit (near the FAST altitude) in the poleward direction. The satellite's trajectory is shown with a dashed line in Fig. 3. The satellite "recorded" oscillations with a period about $0.4 \mathrm{~s}$ and an amplitude of the perpendicular electric field about $3-8 \mathrm{mV} / \mathrm{m}$. These values are close to the parameters of the structures registered by FAST in the EISCAT experiment. Despite this agreement we would avoid to claiming that these particular simulations represent this particular FAST event. First of all, in the experimental event the HF field radiated by the EISCAT high power facility was modulated with a frequency $3 \mathrm{~Hz}$, which is close to the eigenfrequency of the IAR modeled in this paper.

A recent theoretical study by Kolesnikova et al. (2002) demonstrates that the downward fluxes of the accelerated electrons registered by the FAST satellite above the EISCAT heater may be attributed to the parallel electric field in the inertial Alfvén waves directly induced by the heater (Goertz and Boswell, 1979). Estimations given in that paper suggest that Alfvén waves with a transverse wavelength of about $20 \mathrm{~km}$ (i.e. the size of the heated region) would encounter strong inertial dispersion if the background plasma density is about $1 \mathrm{~cm}^{-3}$ at FAST altitude. For more typical values of the density at $2500 \mathrm{~km}$ altitude, about $5 \cdot 10^{2} \mathrm{~cm}^{-3}$ (Kletzing et al., 1998), the transverse wavelength of Alfvén waves should be about $1.5-2 \mathrm{~km}$ to sustain parallel electric field noticeably accelerating electrons (Chaston et al., 2002). Our study demonstrates that these waves can be generated by the ionospheric feedback instability inside the heated region.

Another effect which can further amplify the magnitude of small-scale waves generated inside IAR is the presence of a large-scale density cavity in the direction perpendicular to the ambient magnetic field. The cavity can prevent the resonant wave from leaving the heated region due to their

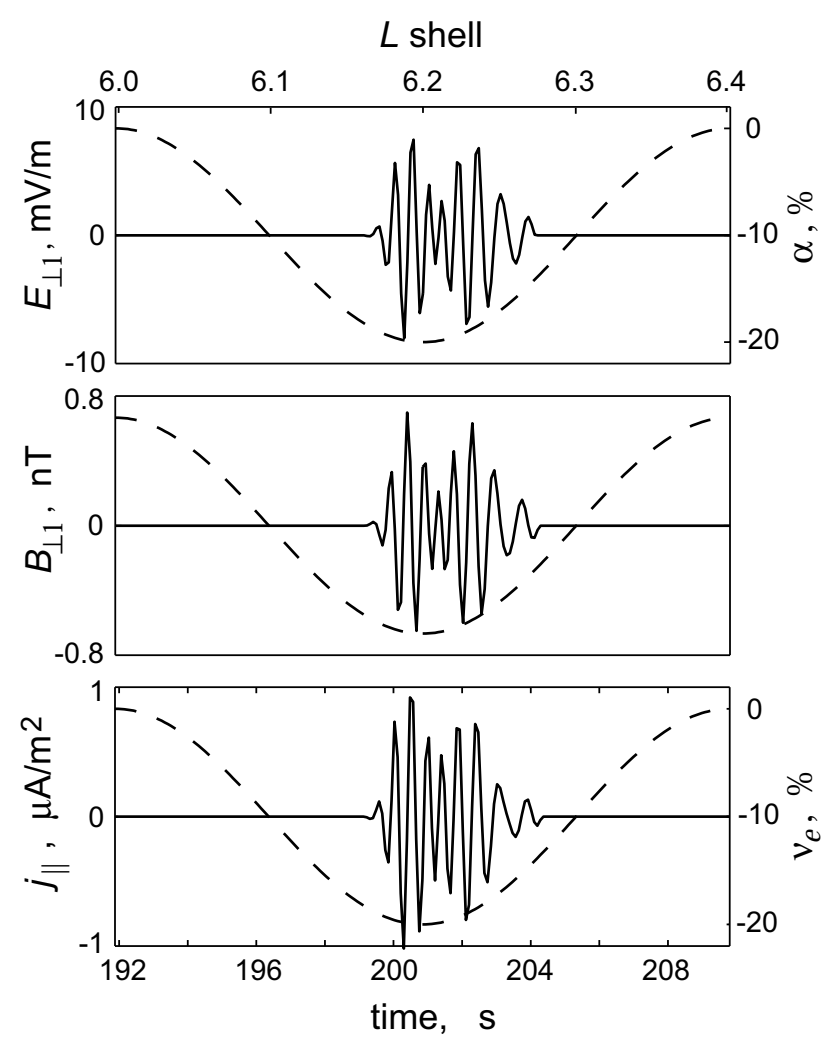

Fig. 4. Solid lines show magnitudes of $E_{1 \perp}, B_{1 \perp}$, and $j_{\|}$observed by the virtual satellite flying poleward across the computational domain at the altitude $2500 \mathrm{~km}$. Dashed lines show spatial variations of $\alpha$ and $v_{e}$ induced by the HF heater in percents of their background value.

high perpendicular phase speed. This trapping mechanism was first discussed by Gul'elmi and Polyakov (1983), and observations from low-orbiting satellites frequently demonstrate intensive, small-scale electromagnetic disturbances inside the density cavities above the auroral and subauroral ionosphere (e.g. Mishin and Förster, 1995; Mishin et al., 2003).

\section{Summary and conclusions}

Numerical simulations of the effects imposed by powerful $\mathrm{HF}$ radio waves have demonstrated that artificial heating may trigger the ionospheric feedback instability when the coupled MI system is close to a state of marginal stability. The effects of artificial heating have been implemented in the numerical model by introducing spatial and temporal variations in the plasma recombination coefficient in the ionospheric E-layer and similar variations in the electron collision frequency in the F-layer. Necessary conditions of marginal stability for the ionospheric feedback have been found using linear dispersion analysis based on the simplified lumped transmission line model of the magnetosphere and upper ionosphere.

Simulation results demonstrate that $20 \%$ variations of the recombination coefficient and electron collision frequency, 
achievable in the ionospheric heating experiments, are sufficient to trigger the ionospheric feedback instability. Development of the instability inside the ionospheric Alfvén resonator leads to the generation of resonant Alfvén waves with the same order of magnitude, though slightly greater than that registered by the FAST satellite during the heating experiment reported by Robinson et al. (2000).

An important limitation of the presented simulation results is the 2-D nature of the numerical model used. In a heating experiment the longitudinal direction of the ionospheric region affected by the HF heater is comparable to its latitudinal size and thus the induced conductivity gradients in longitudes and latitudes can be comparable. As a consequence, the Hall current is expected to influence the ionospheric current closure (Ellis and Southwood, 1983; Yoshikawa and Itonaga, 1996). In particular, one can expect that in the 3-D case ionospheric feedback will generate not only shear Alfvén waves but also compressional waves. That effect can reduce the net power flowing into the feedback-induced Alfvén waves studied in the current 2-D simulations. At the same time we must say that the mode conversion due to the Hall current is a complicated phenomena, depending on a large number of parameters of the waves and the ionosphere and it should be carefully studied with a comprehensive 3-D model. For example, a recent study of the IAR by Pokhotelov et al. (2000) suggests that Hall effects may not be important under some conditions.

\section{Appendix A \\ Dispersion relation for the feedback instability}

A dispersion relation is derived using Eq. (6) that describes lumped transmission line model of the magnetosphere, combined with the linearized ionospheric current Eq. (1) and density Eq. (2) continuity equations. The dispersion relation is expressed by a third-order algebraic equation

$$
A_{3} \omega^{3}+A_{2} \omega^{2}+A_{1} \omega+A_{0}=0,
$$

with the coefficients

$$
\begin{aligned}
& A_{0}=\frac{k_{\perp} M_{P} E_{\perp 0} \pi^{2} v_{A l}^{2}}{\Sigma_{P 0}}+i \frac{2 \alpha n_{0} \pi^{2} v_{A l}^{2}}{\Sigma_{P 0}} \\
& A_{1}=-4 \alpha \mu_{0} l_{0} v_{A l}^{2}-\frac{\pi^{2} v_{A l}^{2}}{\Sigma_{P 0}} \\
& A_{2}=-\frac{k_{\perp} M_{P} E_{\perp 0} l^{2}}{\Sigma_{P 0}}-i\left(2 \mu_{0} l v_{A l}^{2}+\frac{2 \alpha n_{0} l^{2}}{\Sigma_{P 0}}\right) \\
& A_{3}=\frac{l^{2}}{\Sigma_{P 0}} .
\end{aligned}
$$

Here $k_{\perp}$ is the transverse wave number; $\omega=\Omega-i \Gamma$ is the complex frequency, where $\Omega$ and $\Gamma$ denotes the real part of frequency and the growth rate, respectively.

Complex roots of the third-order polynomial Eq. (A1) have been found numerically using Laguerre's method (Press et al., 1992). The numerical solution demonstrates that the only one complex root of dispersion relation Eq. (A1) becomes unstable under certain conditions while two other roots are always stable.

Acknowledgements. Funding for this research has been provided by NASA grants NAG5-10216 and NAG5-11735 (Sun-Earth Connection Theory Program). Computational support was provided by NSF under grant ATM-9977411. The first author (DP) also acknowledges financial support from the Australian Research Council (ARC).

Topical Editor M. Lester thanks E. Hishin and another referee for their help in evaluating this paper.

\section{References}

Atkinson, G.: Auroral arcs: Result of the interaction of a dynamic magnetosphere with the ionosphere, J. Geophys. Res., 75, 4746, 1970.

Blagoveshchenskaya, N. F., Kornienko, V. A., Borisova, T. D., Thidé, B., Kosch, M. J., Rietveld, M. T., Mishin, E. V., Lukyanova, R. Y., and Troshichev, O. A.: Ionospheric HF pump wave triggering of local auroral activation, J. Geophys. Res., 106, $29071,2001$.

Borovsky, J. E.: Auroral arc thicknesses as predicted by various theories, J. Geophys. Res., 98, 6101, 1993.

Chaston, C. C., Bonnell, J. W., Carlson, C. W., Berthomier, M., Prticolas, L. M., Roth, I., McFadden, J. P., Ergun, R. E., and Strangeway, R. J.: Electron acceleration in the ionospheric Alfvén resonator, J. Geophys. Res., 107, 1413, doi:10.1029/2002LA009272, 2002.

Ellis, P. and Southwood, D. J.: Reflection of Alfvén waves by nonuniform ionospheres, Planet. Space Sci., 31, 107, 1983.

Forget, B., Cerisier, J.-C., Berthelier, A., and Berthelier, J.-J.: Ionospheric closure of small-scale Birkeland currents, J. Geophys. Res., 96, 1843, 1991.

Goertz, C. K. and Boswell, R. W.: Magnetosphere-ionosphere coupling, J. Geophys. Res., 84, 7239, 1979.

Gul'elmi, A. and Polyakov, A.: On the discontinous spectrum of Alfvénic oscillations, Geomag. Aeronomy, 23, 281, 1983.

Gurevich, A. V.: Nonlinear phenomena in the ionosphere, Springer Verlag, New York, 370, 1978.

Hardy, D. A., Gussenhoven, M. S., Raistrick, R., and McNeil, W.: Statistical and functional representations of the pattern of auroral energy flux, number flux, and conductivity, J. Geophys. Res., 92, $12275,1987$.

Kelley, M. C.: The Earth's ionosphere, Academic Press, San Diego, 487, 1989.

Kletzing, C. A., Mozer, F. S., and Torbert, R. B.: Electron temperature and density at high latitude, J. Geophys. Res., 103, 14837 , 1998.

Kolesnikova, E., Robinson, T. R., Davies, J. A., Wright, D. M., and Lester, M.: Excitation of Alfvén waves by modulated HF heating of the ionosphere, with applications to FAST observations, Ann. Geophys., 20, 57, 2002.

Lundin, R., Eliasson, L., Haerendel, G., Boehm, M., and Holback, B.: Large-scale auroral plasma density cavities observed by Freja, Geophys. Res. Lett., 21, 1903, 1994.

Lysak, R. L.: Feedback instability of the ionospheric resonant cavity, J. Geophys. Res., 96, 1553, 1991. 
Lysak, R. L. and Song, Y.: Energetics of the ionospheric feedback interaction, J. Geophys. Res., 107, doi:10.1029/2001JA000308, 2002.

Mishin, E. V. and Föerster, M.: "Alfvénic shocks" and low-altitude auroral acceleration, Geophys. Res. Lett., 22, 1745, 1995.

Mishin, E. V., Burke, W. J., Huang, C. Y., and Rich, F. J.: Electromagnetic wave structures within sub-auroral polarization streams, J. Geophys. Res., 108, doi:10.1029/2002JA009793, 2003.

Miura, A. and Sato, T.: Numerical simulation of global formation of auroral arcs, J. Geophys. Res., 85, 73, 1980.

Nygrén, T., Kaila, K. U., and Huuskonen, A.: Determination of E region effective recombination coefficient using impulsive precipitation events, Geophys. Res. Lett., 19, 445, 1992.

Pokhotelov, O. A., Pokhotelov, D., Streltsov, A., Khruschev, V., and Parrot, M.: Dispersive ionospheric Alfvén resonator, J. Geophys. Res., 105, 7737, 2000.

Pokhotelov, D., Lotko, W., and Streltsov, A. V.: Harmonic structure of field line eigenmodes generated by ionospheric feedback instability, J. Geophys. Res., 107, 1363, doi:10.1029/2001JA000134, 2002a.

Pokhotelov, D., Lotko, W., and Streltsov, A. V.: Effects of the seasonal asymmetry in ionospheric Pedersen conductance on the appearance of discrete aurora, Geophys. Res. Lett., 29, 10.1029/2001GL014010, 2002b.

Polyakov, S. V. and Rapoport, V. O.: The ionospheric Alfvén resonator, Geomagn. Aeron., 21, 816, 1981.

Press, W. H., Teukolsky, S. A., Vetterling, W. T., and Flannery, B. P.: Numerical recipes in FORTRAN: the art of scientific computing, Cambridge University Press, Cambridge, New York, 963, 1992.

Rietveld, M. T., Kosch, M. J., Blagoveshchenskaya, N. F., Kornienko, V. A., Leyser, T. B., and Yeoman, T. K.: Ionospheric electron heating, optical emissions, and striations induced by powerful HF radio waves at high latitudes: Aspect angle dependence, J. Geophys. Res., 108, 1141, doi:10.1029/2002JA009543, 2003.

Robinson, T. R., Strangeway, R., Wright, D. M., Davies, J. A., Horne, R. B., Yeoman, T. K., Stocker, A. J., Lester, M., Rietveld, M. T., Mann, I. R., Carlson, C. W., and McFadden, J. P.: FAST observations of ULF waves injected into the magnetosphere by means of modulated HF heating of the auroral electrojet, Geophys. Res. Lett., 27, 3165, 2000.
Sato, T.: A theory of quiet auroral arcs, J. Geophys. Res., 83, 1042, 1978.

Stocker, A. J., Honary, F., Robinson, T. R., Jones, T. B., Stubbe, P., and Kopka, H.: EISCAT observations of large scale electron temperature and electron density perturbations caused by high power HF radio waves, J. Atmos. Terr. Phys., 54, 1555, 1992.

Stocker, A. J., Robinson, T. R., Jones, T. B., and Stubbe, P.: The effects of artificial modifications in the $\mathrm{E}$ region on HF ray propagation, J. Atmos. Terr. Phys., 59, 2435, 1997.

Streltsov, A. V. and Lotko, W.: Dispersive, nonradiative field line resonances in a dipolar magnetic field geometry, J. Geophys. Res., 102, 27 121, 1997.

Streltsov, A. V. and Lotko, W.: Small-scale electric fields in downward auroral current channels, J. Geophys. Res., 108, 1298, doi:10.1029/2002JA009806, 2003.

Streltsov, A. V., Lotko, W., Johnson, J. R., and Cheng, C. Z.: Smallscale, dispersive field line resonances in the hot magnetospheric plasma, J. Geophys. Res., 103, 26 559, 1998.

Stubbe, P., Kopka, H., Lauche, H., Rietveld, M. T., Brekke, A., Holt, O., Jones, T. B., Robinson, T., Hedberg, Å., Thidé, B., Crochet, M., and Lotz, H. J.: Ionospheric modification experiments in northern Scandinavia, J. Atmos. Terr. Phys., 44, 1025, 1982.

Stubbe, P., Kopka, H., Rietveld, M. T., Frey, A., Høeg, P., Kohl, H., Nielsen, E., Rose, G., LaHoz, C., Barr, R., Derblom, H., Hedberg, Å., Thidé, B., Jones, T. B., Robinson, T., Brekke, A., Hansen, T., and Holt, O.: Ionospheric modification experiments with the Troms $\varnothing$ Heating Facility, J. Atmos. Terr. Phys., 47, 1163, 1985.

Trachtengertz, V. Y. and Feldstein, Y. A.: Turbulent Alfvén layer in the polar ionosphere, I, Excitation condition and energetics, J. Geophys. Res., 96, 19363, 1991.

Trachtengertz, V. Y., Belyaev, P. P., Polyakov, S. V., Demekhov, A. G., and Bösinger, T.: Excitation of Alfvén waves and vortices in the ionospheric Alfvén resonator by modulated powerful radio waves, J. Atmos. Sol. Terr. Phys., 62, 267, 2000.

Yoshikawa, A. and Itonaga, M.: Reflection of shear Alfvén waves at the ionosphere and the divergent Hall current, Geophys. Res. Lett., 23, 101, 1996. 